\title{
Existing Difficulties and Solutions for Horizontal Drilling Technique
}

\author{
Wen Ren, Xin Zhang, Hongye Yang, Zhaoting Li, Chunyan Shi \\ CNOOC (China) Co., LTD. Tianjin Branch
}

\begin{abstract}
As most of the main oil fields in the Bohai Oilfield enter the stage of low production, high water content and high recovery, more advanced technology must be adopted to ensure that the Bohai oil fields produce 40 million tons. Based on such a situation, the long horizontal section horizontal well technology came into being, which can achieve a wider range of oilfield exploration, thus greatly enhancing the production of China's oilfields and better serving the people. Although the long horizontal section horizontal well technology has enough advantages to benefit the oilfield industry, however, in the concrete practice, it will encounter many difficult technical aspects of operation. Based on this, this paper provides a brief analysis of the difficulties and countermeasures of long horizontal section horizontal well drilling technology to better apply the technology in the work of producing 40 million tons in Bohai oilfield.
\end{abstract}

Keywords: Horizontal wells, Drilling technology.

\section{Introduction}

In the exploration engineering of the oilfield industry, it is essential to have straight wells and horizontal wells, which can be basically satisfied in the oilfield exploration work in the past because the amount of oilfield is relatively abundant enough, however, with the growth of China's population and the improvement of living standard, the reserve of oilfield resources gradually decreases and increasingly develops to a deeper depth, under such conditions, the general straight wells and horizontal wells Under such conditions, the general straight and horizontal wells can no longer meet the needs of oilfield exploration. Long horizontal section horizontal wells are unique in that they have technical advantages that cannot be surpassed by straight wells and general horizontal wells, and are more capable of exploring deeper and deeper to achieve high production fields. However, in practice, there are many difficulties encountered because of the complex and diverse lithology of the drilling encounters due to the long horizontal section.

\section{Overview of the Horizontal Well Drilling Technology}

At present, in the current situation in China, the long horizontal section horizontal well construction technology is in a rising stage, because of its own technical superiority, many industries will apply the technology to themselves, among them, the most widely applicable industry is the oil extraction and natural gas extraction engineering stage. With the improvement of people's quality of life requirements, the demand for natural gas is also increasing day by day, and the long horizontal section horizontal well construction technology in natural gas extraction engineering shows high technical advantages [1-3].

In thick oil reservoirs, the application of long horizontal section horizontal well technology can achieve more efficient extraction work and greatly increase the amount of extraction, which mainly relies on the seepage area can be significantly increased by long horizontal section horizontal well technology. In addition, in the process of fractured oil reservoirs can also be applied in the long horizontal section horizontal well, and there are outstanding advantages, because ordinary horizontal wells for fractured oil almost no exploration, long horizontal section horizontal well can ensure that the existence of underground fractures remain interconnected and connected, and can carry out effective resource extraction construction; gas top oil and gas reservoirs in the process of extraction using this technology can thoroughly The use of this technology in the process of gas-topped reservoirs can thoroughly deal with the problem of water and gas bubbling in, thus improving the efficiency of extraction.

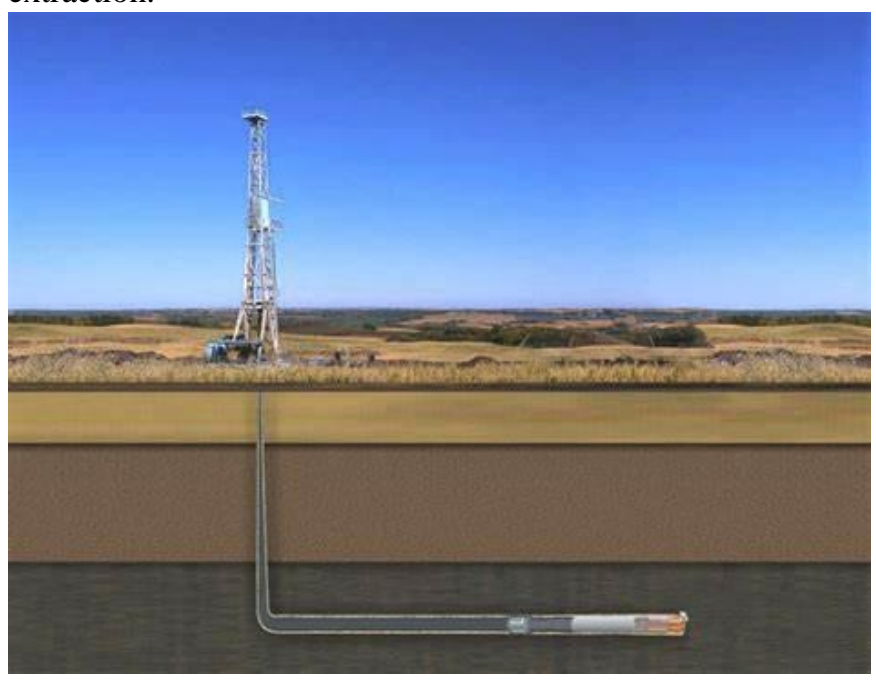

Figure 1: Schematic diagram of horizontal well

\section{The Difficulties of Horizontal Well Drilling Technology}

Exploration work in the oilfield is very important, in order to ensure that the exploration of petroleum projects can achieve more safety and efficient operation, there should be higher requirements for exploration technology, to achieve higher technology exploration. The application of long horizontal section horizontal well technology can greatly realize the amount of exploration and development for the petroleum industry, and is also the main technology application for the 
future petroleum industry. But the technology must be implemented in practice, long horizontal section horizontal well in the practical application will have many aspects of the difficulties, mainly because the process is cumbersome, for the equipment parameters are also higher requirements, capital investment efforts, only the long horizontal section horizontal well better applied to the oil industry exploration project, which is also the current inevitable development situation of China's petroleum industry. Mainly from the following aspects of the long horizontal section horizontal well drilling technology application difficulties to analyze.

\subsection{Drilling Trajectory is Difficult to Control}

In the past, the technical application for oil exploration is mainly since the horizontal and straight wells, in the specific actual crossing the number of underground target points is generally limited and fixed, so that according to the number of target points can accurately determine the trajectory of the extraction process, for the drilling project to reduce the implementation difficulties and achieve effective control [4]. However, the long horizontal section horizontal well drilling technology is to achieve a wider area and deeper exploration, which requires that the number of targets traversing in the subsurface will increase a lot, thus causing the drilling trajectory in the mining process can not be determined, without a clear trajectory, the rest of the mining work can not be started, if the trajectory because of the Errors in deviation may cause the actual quantity of exploration in the field to be unrealized [5]. Therefore, the difficulty in controlling the drilling trajectory is one of the most prominent technical difficulties in long horizontal section horizontal wells, and more problems can arise during the drilling process, such as stuck drilling. Another aspect is that the difficulty is also higher when there are more types of formation rocks and unevenly distributed sections.

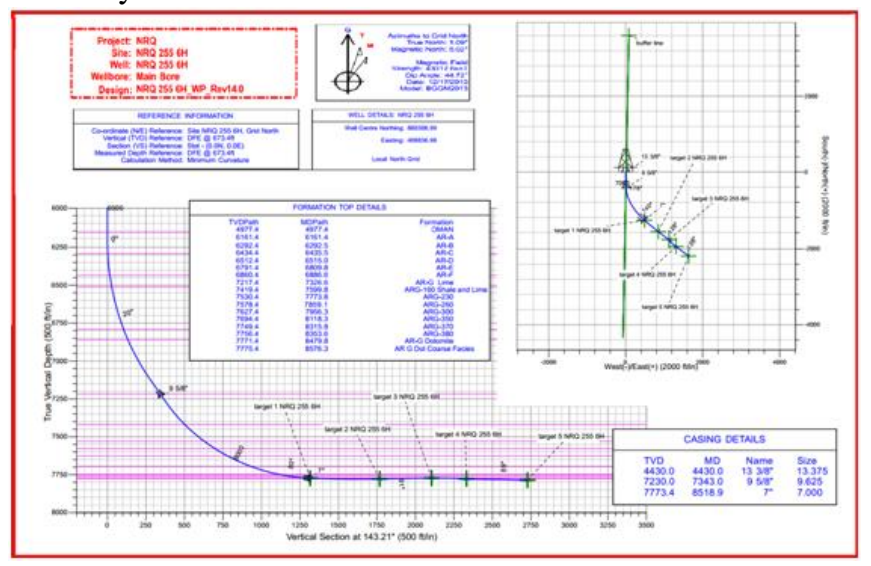

\subsection{Drilling Friction is Too Large}

Long horizontal section horizontal well drilling technology can achieve greater penetration area of exploration and mining, the realization of a large area will inevitably rely on a larger area of contact with the ground, the contact area of the drill bit, drill pipe and drilling wall will be larger than other technologies, the contact area will cause greater resistance in the process of drilling technology, because the resulting frictional resistance is greater, making the drilling speed slow down, it is difficult to achieve in It is difficult to complete the drilling of long horizontal section horizontal wells within the specified time frame [6]. In addition, long horizontal section horizontal well drilling technology in the specific implementation of the project, the underground distance is very long, which will also lead to the degree and amount of underground rock damage will increase, drilling fluid directly from the wellhead is difficult to achieve, the lack of drilling fluid, drilling in the process of lubrication will be weakened, the resistance will also be correspondingly increased, bringing greater difficulty for the project, the follow-up work can not be carried out The subsequent work cannot be carried out.

\subsection{Difficult to Lower Casing}

In the process of applying long horizontal section horizontal well drilling technology, because the slope rate in drilling is larger than other technologies, in the stage of casing construction, the contact area between the casing and the wall will be larger, which leads to an increase in friction, and the difficulty of casing construction will be increased.

\subsection{Difficulty in Curing Wells}

Cementing in oil exploration drilling technology is a very critical step, because after the completion of the horizontal well will immediately begin to cementing treatment, if not immediately processed will make the initial drilling work in vain or the subsequent work can not be normal and effective smoothly. For the construction of long horizontal section horizontal well drilling, the inseparable raw material is cement paste, and the ordinary drilling technology does not have high requirements for the quality of cement paste, but the long horizontal section horizontal well drilling technology has higher requirements for the quality of cement paste. However, in the actual project, because the inclined and horizontal sections are problematic, the cementing quality of the cement slurry is also poor, which leads to the influence of the well wall quality [7] and the quality of cementing cannot be guaranteed.

\section{Long Horizontal Section Horizontal Well Drilling Technical Difficulties Control Measures}

The above is mainly an analysis of the long horizontal section horizontal well drilling technology in the actual project encountered technical difficulties, and these technical difficulties are basically more common, but also key, only breakthrough technical difficulties, in order to achieve a more practical application of long horizontal section horizontal well drilling technology, really become the core technology of the oil industry exploration and exploitation, the following technical difficulties, pointed out the long horizontal section horizontal well drilling technology difficult to solve measures to improve the application of long horizontal section horizontal well drilling technology.

\subsection{Drilling Trajectory Control Measures}


In order to completely solve the problem of difficult to control the drilling trajectory, firstly, the specific location of the underground target point should be accurately determined, and then the specific quantity should be clarified; secondly, before the drilling starts, the characteristics of the underground rocks should be understood in detail, and the slope making construction should be carried out at the suitable location; finally, in the drilling construction stage, the suitable reversing drilling tools should be selected so that the drilling trajectory can be accurately controlled [8].

\subsection{Countermeasures for Excessive Drilling Resistance}

In order to completely eliminate the problem of excessive friction, firstly, the value of friction parameters should be accurately evaluated before drilling starts, and then suitable drilling tools should be selected for construction according to this data; secondly, the combination of drilling tools should be continuously optimized, and it is better to choose the structure form of sloping drill pipe, whose main advantage is that the contact area is lower, which can greatly reduce the friction; finally, a layer of lubricating substance should be painted on the outer surface of the drilling tools, which can reduce the friction [9].

\subsection{Countermeasures Against the Difficulty of Lowering Casing}

In the process of drilling construction, the structure should be reasonably designed, and the well construction should be done well to prevent the problem of stalling during the construction; the casing construction stage should be divided into two or more sections to carry out, which can effectively Reduce friction. Keep the structure smooth and make the casing construction more smooth.

\subsection{Difficult Countermeasures for Cementing}

In the drilling construction project, this part of the cementing is very critical, but because the inclined and horizontal sections are problematic, the cementing quality of the cement paste is poor, which leads to the existence of the impact of the quality of the well wall. For this problem, the most effective and main solution is to improve the stability of the well wall, stability is greater, the cementing project will be more convenient in the specific implementation process. To improve the stability of the well wall is first of all related to the drilling fluid, therefore, according to the basis of the subsurface pressure to choose the appropriate drilling fluid, and according to the actual situation of the project to match the mud; of course, the trajectory of the drilling construction is to fully understand, in order to solve the excessive drilling friction with the basis of the lower pipe to ensure the successful completion of the cementing.

\section{Conclusion}

In summary, the oil industry has become one of the focus of society today, oil is widely used in people's daily lives, in order to ensure the quantity and management quality of oil, the technical requirements for its exploration and extraction process has a higher demand. The current long horizontal section horizontal well drilling technology is an important driving force for oil exploration and extraction projects, and an important measure to ensure the safety of oil reservoirs. The application of long horizontal section horizontal well drilling technology in China's oil extraction field can greatly improve the extraction efficiency and extraction quality, but there are many difficulties and problems in the construction, it is necessary to strengthen the research and development and application of technology to completely solve the technical difficulties, in order to can ensure the development of China's oil extraction business.

\section{References}

[1] Chemical Industry Management,2021(15):62-63. Zhu Yulei. Technical difficulties and strategies for drilling extended reach horizontal Wells [J]. Chemical Industry Management, 2021(15): 62-63.

[2] Li Fan. Application of new production logging technology for horizontal well in offshore oilfield [J]. China Petroleum \& Chemical Standards and Quality, 2011, 41(07): 163-164.

[3] Wu Beibei, Zhang Fei, Chang Ziheng, Sun Yuanzhen, Li Peng. Technical difficulties and countermeasures of drilling horizontal Wells for natural gas in Yanchang Gas Field [J]. Yunnan Chemical Industry, 2013, 48(04):172-174.

[4] Wu Guochao. Application of horizontal well drilling technology in A block of D oilfield [J]. West China Exploration Engineering, 2010, 33(05):92-94.

[5] Chemical Engineering and Equipment,2021(04):65-66. LI Min. Method of improving fine geology of horizontal well $[\mathrm{J}]$. Chemical Engineering and Equipment, 2021(04): 65-66.

[6] Gao Zhixing. Research and application of horizontal well cementing technology [J]. Petroleum and Chemical Equipment, 2016, 24(04): 112-114.

[7] CAO Zhiyu. Research on Factors Affecting Friction and Friction Reduction Technology of Horizontal Well Drilling [J]. China Petroleum \& Chemical Standards and Quality, 2019, 41(05):24-26.

[8] Yuan Xiwang. Discussion on the application of underbalanced drilling technology in long horizontal well [J]. West China Exploration Engineering, 2019, 33(04): 93-94+96.

[9] Li Pengfei, Yao Chunyu, Chen Chuan. Analysis of precise control technology of horizontal well trajectory [J]. Chemical Industry Management, 2021(03): 189-190. 